\title{
Low polymorphism revealed in new microsatellite markers for Bemisia tabaci (Hemiptera: Aleyrodidae)
}

\author{
G.E. Valle ${ }^{1}$, A.L. Lourenção ${ }^{1}$, M.I. Zucchi ${ }^{3}$ and J.B. Pinheiro ${ }^{2}$ \\ ${ }^{1}$ Seção de Entomologia, Centro de Fitossanidade, \\ Instituto Agronômico de Campinas, Campinas, SP, Brasil \\ 2Departamento de Genética, Escola Superior de Agricultura "Luiz de Queiroz", \\ Universidade de São Paulo, Piracicaba, SP, Brasil \\ ${ }^{3}$ Agência Paulista de Tecnologia dos Agronegócios, \\ Pólo Centro Sul, Piracicaba, SP, Brasil \\ Corresponding author: A.L. Lourenção \\ E-mail: andre@iac.sp.gov.br
}

Genet. Mol. Res. 11 (4): 3899-3903 (2012)

Received May 10, 2012

Accepted July 19, 2012

Published November 12, 2012

DOI http://dx.doi.org/10.4238/2012.November.12.7

\begin{abstract}
The silverleaf whitefly (Bemisia tabaci) is actually a complex of morphologically indistinct species that vary in their capacity to transmit plant viruses and to induce physiological disorders in plants of economic importance. The worldwide impact of this whitefly has increased greatly, as it is a vector of various types of phytovirus, especially geminiviruses, in plants of economic importance. The adaptability of $B$. tabaci to many regions of the world has fostered the appearance of various biotypes that attack a broad spectrum of host plants. We developed microsatellite markers to study genetic variability and population structure of this whitefly in Brazil. Thirteen polymorphic microsatellite markers were isolated and characterized in 20 individuals from a natural population that were collected in soybean in Campinas (SP). The number of alleles per locus ranged from one to two, and the expected heterozygosity ranged from 0.000 to 0.505 . These microsatellite
\end{abstract}


markers will be useful for studies and management of B. tabaci. The low polymorphism found in these molecular markers is probably associated with homology of genes expressed in these markers.

Key words: Silverleaf whitefly; Homology; Molecular markers; Monomorphic microsatellite; Whitefly

\section{INTRODUCTION}

More than 1500 whitefly species (Hemiptera: Aleyrodidae) are known in the whole world (Martin and Mound, 2007); however, very few of these insects have been studied more specifically (Byrne et al., 1990). Bemisia tabaci (Genn.) is one of the most important species, considered as a complex (Brown et al., 1995; Perring, 2001) and has a wide spectrum of hosts. B. tabaci develops in more than 900 host plants (Perring, 2001; Berry et al., 2004), where it is responsible for more than 110 different viruses and transmitting geminivirus (Jones, 2003). Therefore, the objective of the present research was to develop microsatellite molecular markers through genomic-enriched library protocols for future studies of genetic variability, population structure and gene flow of $B$. tabaci.

\section{MATERIAL AND METHODS}

Genomic DNA was extracted from a whitefly female using an extraction protocol described by Lima et al. (2000). Only one whitefly female was macerated in $60 \mu \mathrm{L}$ extraction buffer $(1 \mathrm{mM}$ Tris-HCl, $\mathrm{pH}$ 8.0, $5 \mathrm{mM} \mathrm{NaCl}, 1 \mathrm{mM}$ EDTA, $\mathrm{pH}$ 8.0, 3\% Triton X-100, $60 \mu \mathrm{g} / \mathrm{mL}$ proteinase $\mathrm{K}$ ). The macerate was incubated for $15 \mathrm{~min}$ at $65^{\circ} \mathrm{C}$, followed by heating for $10 \mathrm{~min}$ at $95^{\circ} \mathrm{C}$. A microsatellite-enriched library was obtained using adapted protocols from Billotte et al. (1999). Genomic DNA from one genotype of B. tabaci was digested with AfaI (Invitrogen) and enriched in microsatellite fragments using $(\mathrm{CT})_{8}$ and $(\mathrm{GT})_{8}$ motifs. The enriched fragments were cloned into pGEM77 T (Promega) and ligation products were used to transform Epicurian Coli XL1-Blue Escherichia coli competent cells. The positive clones were selected using the $\beta$-galactosidase gene and then grown overnight in the presence of ampicillin. A total of 117 clones were sequenced in an ABI 3770 automated 79 sequencer (PE Applied Biosystems) using a BigDye terminator cycle sequencing kit (Applied Biosystems). About 10 primer pairs were designed for SSR flanking regions using Primer 3 (Rozen and Skaletsky, 2000) and tested in 20 specimens of B. tabaci collected from different regions in Brazil. PCRs were performed in a $20-\mu \mathrm{L}$ reaction volume containing $1 \mathrm{X}$ reaction buffer $(100 \mathrm{mM}$ Tris- $\mathrm{HCl}, \mathrm{pH} 8.5,50 \mathrm{mM} \mathrm{KCl})$ containing $20 \mathrm{ng}$ genomic DNA, $0.2 \mu \mathrm{M}$ of each primer, $0.25 \mathrm{mM}$ dNTPs, $0.5 \mathrm{U}$ Taq DNA polymerase, and $4 \mathrm{mM}$ $\mathrm{MgCl}_{2}$. The PCR program consisted of an initial denaturing step at $95^{\circ} \mathrm{C}$ for 2 min followed by 45 cycles of amplification $\left[95^{\circ} \mathrm{C}(1 \mathrm{~min}), 1 \mathrm{~min}\right.$ at the specific annealing temperature of each primer pair (Table 1), and $72^{\circ} \mathrm{C}(1 \mathrm{~min})$ ], and a final elongation step at $72^{\circ} \mathrm{C}$ for $5 \mathrm{~min}$. Amplification products were resolved by electrophoresis on $7 \%$ denaturing polyacrylamide gels and visualized by silver staining (Creste et al., 2001). The allele scoring was done using the 10-bp DNA Ladder (Invitrogen) for size standards. The population's parameters of the 13 loci obtained were estimated by using the Genetix 4.05.2 program (Belkhir et al., 2004) and by genotyping 20 individuals of $B$. tabaci from a natural population collected in Campinas, SP, Brazil. 
Table 1. Characteristics of 13 microsatellite loci from Bemisia tabaci.

\begin{tabular}{|c|c|c|c|c|c|c|c|c|c|}
\hline Locus & Repeat motif & Primer sequence (5'-3') & $\mathrm{Ta}\left({ }^{\circ} \mathrm{C}\right)$ & Size range (bp) & $\mathrm{N}$ & $H_{\mathrm{O}}$ & $H_{\mathrm{E}}$ & PIC & $\begin{array}{c}\text { GenBank } \\
\text { accession No. }\end{array}$ \\
\hline Bta 1 & $(\mathrm{CTT})_{4}$ & $\begin{array}{l}\text { F: TGAAGGAGACTTGGCAACG } \\
\text { R: ATCTCTCTCACCGCCATCAT }\end{array}$ & 62.8 & 224 & 2 & 0.000 & 0.337 & 0.269 & GF111984 \\
\hline Bta2 & $(\mathrm{TACC})_{3}$ & $\begin{array}{l}\text { F: AAAAGTCGTCCGCTGTTACC } \\
\text { R: CGGGGAAATAAAAGCAACTG }\end{array}$ & 63.8 & 177 & 1 & 0.000 & 0.000 & 0.000 & GF111977 \\
\hline Bta3 & $(\mathrm{GAAA})_{3}$ & $\begin{array}{l}\text { F: TATACTTGGGCATCGTCAG } \\
\text { R: GCGTAGGAGAGTTGGAATG }\end{array}$ & 57.7 & 228 & 1 & 0.000 & 0.000 & 0.000 & GF111978 \\
\hline Bta4 & $(\mathrm{TTTA})_{3}$ & $\begin{array}{l}\text { F: CGCTCCTCAAGTTTTCTGT } \\
\text { R: CGGCAGTCAGGGTTATTA }\end{array}$ & 58.9 & 175 & 2 & 0.000 & 0.442 & 0.332 & GF111985 \\
\hline Bta5 & $(\mathrm{AAAG})_{3}$ & $\begin{array}{l}\text { F: TATACTTGGGCATCGTCAGC } \\
\text { R: GCGTAGGAGAGTTGGAATGC }\end{array}$ & 62.7 & 228 & 1 & 0.000 & 0.000 & 0.000 & GF111979 \\
\hline Bta6 & $(\mathrm{AATA})_{3}$ & $\begin{array}{l}\text { F: TTGTTGGTTCACCTGTAATTGG } \\
\text { R: GCGTGGACTAGCTGAAAAAGA }\end{array}$ & 64.7 & 150 & 2 & 0.000 & 0.505 & 0.365 & GF111980 \\
\hline Bta7 & $(\mathrm{TTCT})_{4}$ & $\begin{array}{l}\text { F: CTGTAACGGACACACACACA } \\
\text { R: GGTCATTTTGGTGTGAA }\end{array}$ & 58.1 & 244 & 1 & 0.000 & 0.000 & 0.000 & GF111986 \\
\hline Bta11 & $(\mathrm{TCAT})_{3}$ & $\begin{array}{l}\text { F: TTTTTCGGTTGGTTGGTG } \\
\text { R: GCTAATTGCTTCTTTCCTTG }\end{array}$ & 60.8 & 155 & 1 & 0.000 & 0.000 & 0.000 & GF111987 \\
\hline Bta12 & $(\mathrm{AAAT})_{6}$ & $\begin{array}{l}\text { F: TCCCATCTCCGCCTTATCTA } \\
\text { R: GACCTCCGCAAGTTTTTCC }\end{array}$ & 64.1 & 166 & 2 & 0.000 & 0.505 & 0.365 & GF111981 \\
\hline Bta13 & $\begin{array}{l}(\mathrm{CT})_{12}(\mathrm{CT})_{8} \\
(\mathrm{CACT})_{3}(\mathrm{CT})_{7}\end{array}$ & $\begin{array}{l}\text { F: AAACGTGGTCCCTTGGAATA } \\
\text { R: AACGCCCTTTCACAATTCA }\end{array}$ & 63.8 & 248 & 1 & 0.000 & 0.000 & 0.000 & GF111982 \\
\hline Bta16 & $(\mathrm{TCAT})_{3}$ & $\begin{array}{l}\text { F: GGCTAATTGCTTCTTTCCTTG } \\
\text { R: GTTTTTCGGTTGGTTGGTG }\end{array}$ & 63.8 & 155 & 1 & 0.000 & 0.000 & 0.000 & GF111988 \\
\hline Bta17 & $(\mathrm{TACG})_{3}$ & $\begin{array}{l}\text { F: CACCCTCACCTACACACACAC } \\
\text { R: TCACAACAAATGCGACCTT }\end{array}$ & 61.6 & 206 & 1 & 0.000 & 0.000 & 0.000 & GF111983 \\
\hline Bta18 & $(\mathrm{TCAT})_{3}$ & $\begin{array}{l}\text { F: GGTGGTTTGTTTAGAAGAGTGG } \\
\text { R: GGCTAATTGCTTCTTTCCTTG }\end{array}$ & 63.0 & 193 & 1 & 0.000 & 0.000 & 0.000 & GF111989 \\
\hline
\end{tabular}

$\mathrm{F}$ = forward primer sequence; $\mathrm{R}=$ reverse primer sequence; $\mathrm{Ta}=$ annealing temperature; $\mathrm{N}=$ number of alleles; $H_{\mathrm{O}}=$ observed heterozygosity; $H_{\mathrm{E}}=$ expected heterozygosity; PIC = polymorphic information content.

\section{RESULTS AND DISCUSSION}

From the library, 344 clones were sequenced and 103 SSRs were found: $17.5 \%$ mononucleotides, $33.0 \%$ dinucleotides, $0.9 \%$ trinucleotides, $15.5 \%$ tetranucleotides, $8.8 \%$ pentanucleotides, and $24.3 \%$ compounds. Of these sequences, 13 had a flanking region of adequate size for the design of forward and reverse primers. The number of alleles observed for each locus ranged from one to two. We found a low level of polymorphism among 20 individuals analyzed with the new locus developed. Thirteen loci were developed but only four were polymorphic with two alleles per locus. We showed a low level of polymorphism in the genotypes evaluated. The expected heterozygosity ranged from 0.000 to 0.505 . For all loci, the observed heterozygosity was lower than or equal to the expected heterozygosity. The low polymorphism found in these molecular markers is probably associated with homology to the genes expressed in all these markers (Table 2). The studies of genetic variability of insect pests, in the context of insect resistance management, can provide useful information about distribution patterns of genetic variability and gene flow that allow the determination of focus areas for monitoring and the development of strategies to mitigate the development of resistance. Obtaining specific primers that access microsatellite loci in $B$. tabaci enables the study of this genetic variability and generates information that could be used in future management programs of this pest. A previous study with other molecular markers (SSRs) for B. tabaci also showed a low level of polymorphism (Valle et al., 2011). A recent tool for genetic analysis was developed by Nazereno and Reis (2011), which consisted in evaluating monomorphic 
microsatellites; therefore, in new and future studies, these analyses will be used to determine the reason for the low polymorphism found in this study.

Table 2. Homology of 13 microsatellite loci from Bemisia tabaci.

\begin{tabular}{|c|c|c|c|c|}
\hline Primer & Homology & GenBank accession No. & E value & Max ident (\%) \\
\hline \multirow[t]{2}{*}{$\overline{B t a 1}$} & Rat alpha-amylase (Amy-1) gene, 5'-end, and parotid-specific promoter region & M14151.1 & 0.002 & 90 \\
\hline & Mus musculus mRNA for mKIAA0164 protein & AK129071.1 & 0.020 & 90 \\
\hline \multirow[t]{2}{*}{ Bta2 } & Fragaria $\mathrm{x}$ ananassa gene encoding methionine sulfoxide reductase & AJ297967.1 & $5 e-10$ & 83 \\
\hline & Gallus gallus mRNA for hypothetical protein, clone 22014 & AJ720655.1 & $5 \mathrm{e}-10$ & 83 \\
\hline \multirow[t]{3}{*}{ Bta3 } & Uncultured archeon partial 16S rRNA gene, isolate AEXO15n.4 & FM242736.1 & $2 \mathrm{e}-18$ & 84 \\
\hline & Uncultured bacterium partial 16S rRNA gene, isolate EXO15432 & FM242723.1 & $2 \mathrm{e}-18$ & 84 \\
\hline & $\begin{array}{l}\text { Bacterium AK-MB19 partial yfkC gene for putative reverse } \\
\text { transcriptase maturase protein, isolate MB } 19\end{array}$ & AM997282.1 & $3 e-15$ & 81 \\
\hline \multirow[t]{3}{*}{$\mathrm{Bta} 4$} & Fragaria $\mathrm{x}$ ananassa gene encoding methionine sulfoxide reductase & AJ297967.1 & $5 e-10$ & 83 \\
\hline & Gallus gallus mRNA for hypothetical protein, clone 22014 & AJ720655.1 & $5 e-10$ & 83 \\
\hline & $\begin{array}{l}\text { Arabidopsis lyrata clone MITE7 transposon-insertion display } \\
\text { band genomic sequence }\end{array}$ & EU558532.1 & $6 \mathrm{e}-09$ & 82 \\
\hline \multirow[t]{4}{*}{ Bta5 } & Macrococcus caseolyticus JCSC5402 DNA, complete genome & AP009484.1 & 0.056 & 83 \\
\hline & Paramecium tetraurelia hypothetical protein (GSPATT00012894001) partial mRNA & XM001444769.1 & 0.20 & 83 \\
\hline & $\begin{array}{l}\text { Oryzias marmoratus PSMB } 10 \text {, PSMB } 8 \text { genes for proteasome subunit, } \\
\text { beta type } 10 \text {, proteasome subunit, beta type } 8 \text {, partial and complete cds, } \\
\text { isolate: Oryzias marmoratus } 15\end{array}$ & AB551024.1 & 0.68 & 93 \\
\hline & $\begin{array}{l}\text { Oryzias matanensis PSMB10, PSMB8 genes for proteasome subunit, } \\
\text { beta type } 10 \text {, proteasome subunit, beta type } 8 \text {, partial and complete cds, } \\
\text { isolate: Oryzias matanensis } 6 \text {, haplotype: } \mathrm{N}\end{array}$ & AB551021.1 & 0.68 & 93 \\
\hline \multirow[t]{3}{*}{ Bta6 } & Bemisia tabaci isolate Mogi Guaçu haplotype 4 cytochrome oxidase subunit & JN689359.1 & $7 e-94$ & 100 \\
\hline & Bemisia tabaci isolate Sumare haplotype 3 cytochrome oxidase subunit & JN689358.1 & $7 \mathrm{e}-94$ & 100 \\
\hline & $\begin{array}{l}\text { Bemisia tabaci isolate Ilha Solteira haplotype } 2 \text { cytochrome oxidase } \\
\text { subunit I (COI) gene, partial cds; mitochondrial }\end{array}$ & JN689357.1 & $7 \mathrm{e}-94$ & 100 \\
\hline \multirow[t]{6}{*}{ Bta7 } & Sorghum bicolor hypothetical protein, mRNA & XM002441933.1 & $4 \mathrm{e}-07$ & 85 \\
\hline & Solanum lycopersicum goblet gene, complete cds & HM210879.1 & $3 e-08$ & 85 \\
\hline & Picea glauca clone GQ03118 G07 mRNA sequence & BT108209.1 & $1 \mathrm{e}-08$ & 86 \\
\hline & Petunia hybrida NAM gene ${ }^{-}$ & X92204.1 & $4 \mathrm{e}-07$ & 82 \\
\hline & Zea mavs clone 345601 CUC2 mRNA, complete cds & EU970442.1 & $1 \mathrm{e}-06$ & 84 \\
\hline & Amborella trichopoda mRNA for the NAM protein & FR668080.1 & $5 e-06$ & 83 \\
\hline \multirow[t]{7}{*}{ Bta11 } & Solanum tuberosum no apical meristem (NAM) mRNA, complete cds & FJ435166.1 & $3 e-09$ & 89 \\
\hline & Picea glauca clone GQ03118 G07 mRNA sequence & BT108209.1 & $1 \mathrm{e}-08$ & 86 \\
\hline & Sorghum bicolor hypothetical protein, mRNA & XM002462559.1 & $3 \mathrm{e}-08$ & 86 \\
\hline & Zea mays partial mRNA for hypothetical protein (nam1 gene) & AJ833966.1 & $4 \mathrm{e}-07$ & 86 \\
\hline & Amborella trichopoda mRNA for the NAM protein & FR668080.1 & $5 \mathrm{e}-06$ & 86 \\
\hline & Brassica oleracea cup-shaped cotyledon 2 (CUC2) gene, partial cds & HQ703968.1 & $2 \mathrm{e}-04$ & 87 \\
\hline & Ginkgo biloba partial mRNA for the NACa protein & FR668082.1 & $2 \mathrm{e}-04$ & 87 \\
\hline \multirow[t]{5}{*}{ Bta12 } & Mus musculus chromosome 5, clone RP24-465G18, complete sequence & AC133456.11 & $7 e-16$ & 87 \\
\hline & Mus musculus chromosome 5 , clone RP23-37G13, complete sequence & AC161225.14 & $7 \mathrm{e}-16$ & 87 \\
\hline & Homo sapiens X BAC RP11-126O22 (Roswell Park Cancer Institute & AC073488.23 & $1 \mathrm{e}-11$ & 93 \\
\hline & Human BAC Library) complete sequence & & & \\
\hline & Human T-cell receptor alpha delta locus, complete sequence & AE000521.1 & $1 \mathrm{e}-05$ & 88 \\
\hline \multirow[t]{3}{*}{ Bta13 } & Bacteriovorax marinus SJ genome & FQ312005.1 & 4.3 & 89 \\
\hline & Gossypium hirsutum TM-1 D-subgenome 14-3-3-like protein (14-3-3L & HQ142993.1 & 4.3 & 88 \\
\hline & $\begin{array}{l}\text { Karlodinium micrum clone } 17645 \text { cytochrome oxidase subunit III } \\
\text { (COX3) mRNA, partial cds; mitochondrial }\end{array}$ & EF443024.1 & 0.35 & 81 \\
\hline \multirow[t]{2}{*}{ Bta16 } & Zebrafish DNA sequence from clone DKEY-246M18 in linkage group 15 & BX571794.22 & $1 \mathrm{e}-05$ & 73 \\
\hline & $\begin{array}{l}\text { PREDICTED: Acyrthosiphon pisum tyrosine-protein phosphatase } \\
\text { non-receptor type 21-like (LOC100161359), mRNA }\end{array}$ & XM001951853.2 & $2 \mathrm{e}-20$ & 84 \\
\hline \multirow[t]{7}{*}{ Bta17 } & $\begin{array}{l}\text { Homo sapiens N-acetylglucosamine-1-phosphate transferase, alpha and } \\
\text { beta subunits (GNPTAB), RefSeqGene on chromosome } 12\end{array}$ & NG021243.1 & 0.38 & 85 \\
\hline & $\begin{array}{l}\text { Karlodinium micrum clone } 1939 \text { cytochrome oxidase subunit III } \\
\text { (COX3) mRNA, partial cds; mitochondrial }\end{array}$ & EF443026.1 & 0.38 & 81 \\
\hline & Homo sapiens KIAA0438 mRNA, partial cds & AB007898.1 & 1.3 & 96 \\
\hline & Pinellia cordata voucher T.S. Yi $08015 \mathrm{rps} 16$ gene, intron; chloroplast & JQ237171.1 & 4.6 & 90 \\
\hline & Arcobacter sp L DNA, complete genome & AP012048.1 & 4.6 & 81 \\
\hline & Puccinia graminis $\mathrm{f}$. $\mathrm{sp}$ tritici $\mathrm{CRL} 75-36-700-3$ hypothetical protein, mRNA & XM003319919.1 & 4.6 & 84 \\
\hline & $\begin{array}{l}\text { Gossypium hirsutum TM-1 D-subgenome 14-3-3-like protein } \\
\text { (14-3-3L) gene, complete CDs }\end{array}$ & HQ142993.1 & 4.6 & 88 \\
\hline \multirow[t]{3}{*}{ Bta18 } & Flavobacterium columnare ATCC 49512, complete genome & СР003222.2 & 0.11 & 79 \\
\hline & $\begin{array}{l}\text { Solanum lycopersicum strain Heinz } 1706 \text { chromosome } 1 \text { clone } \\
\text { slm- } 15 \mathrm{p} 5 \text { map } 1 \text {, complete sequence }\end{array}$ & AC246100.4 & 0.38 & 85 \\
\hline & $\begin{array}{l}\text { Gossypium hirsutum TM-1 D-subgenome 14-3-3-like protein } \\
\text { (14-3-3L) gene, complete cds }\end{array}$ & HQ142993.1 & 4.6 & 88 \\
\hline
\end{tabular}




\section{REFERENCES}

Belkhir K, Borsa P, Chikhi L, Raufaste N, et al (2004). GENETIX 4.05, Logiciel Sous Windows TM Pour la Génétique des Populations. Laboratoire Génome, Populations, Interactions, CNRS UMR 5000, Université de Montpellier II, Montpellier. Available at [http://www.genetix.univ-montp2.fr/genetix/genetix.htm/] Accessed September 9, 2010.

Berry SD, Fondong VN, Rey C, Rogan D, et al. (2004). Molecular evidence for five distinct Bemisia tabaci (Homoptera: Aleyrodidae) geographic haplotypes associated with cassava plants in Sub Saharan Africa. Ann. Entomol. Soc. Am. 97: 852-859.

Billotte N, Lagoda PJR, Risterucci AM and Baurens FC (1999). Microsatellite-enriched libraries: applied methodology for the development of SSR markers in tropical. Crops Fruits 54: 277-288.

Brown JK, Frohlich DR and Rosell RC (1995). The sweetpotato or silverleaf whiteflies: biotypes of Bemisia tabaci or a species complex? Ann. Rev. Entomol. 40: 511-534.

Byrne DN, Bellows TS Jr and Parella MP (1990). Whiteflies in Agricultural Systems. In: Whiteflies: Their Bionomics, Pest Status, and Management (Gerling D, ed.). Intercept Ltd., Andover, 227-261.

Creste S, Tulmann Neto A and Figueira A (2001). Detection of single repeat polymorphism in denaturing polyacrylamide sequencing gels by silver staining. Plant Mol. Biol. Rep. 19: 299-306.

Jones DR (2003). Plant viruses transmitted by whiteflies. Eur. J. Plant Pathol. 109: 195-219.

Lima LHC, Návia D, Inglis PW and Oliveira MRV (2000). Survey of Bemisia tabaci (Gennadius) (Hemiptera: Aleyrodidae) biotypes in Brazil using RAPD markers. Genet. Mol. Biol. 23: 781-785.

Martin JH and Mound LA (2007). An annotated check list of the world's whiteflies (Insecta: Hemiptera: Aleyrodidae). Zootaxa 1492: 1-84.

Nazareno AG and Reis MS (2011). The same but different: monomorphic microsatellite markers as a new tool for genetic analysis. Am. J. Bot. 98: e265-e267.

Perring TM (2001). The Bemisia tabaci species complex. Crop Prot. 20: 725-737.

Rozen S and Skaletsky HJ (2000). Primer3 on the WWW for general users and for biologist programmers. In: Bioinformatics Methods and Protocols: Methods in Molecular Biology (Krawetz and Misener S, eds.). Humana Press, Totowa, 365-386.

Valle GE, Zucchi MI, Stabellini NS, Lourenção AL, et al. (2011). Estrutura genética populacional de Bemisia tabaci (Gennadius) (Hemiptera: Aleyrodidae) utilizando marcadores microssatélites. Neotrop. Entomol. 40: 204-211. 